\title{
The Imiquimod Induced Psoriatic Animal “Model: Scientific Implications
}

\author{
Banzragch Dorjsembe ${ }^{1,2}$, Jung Yeob Ham ${ }^{1}$ and Jin Chul Kim*1 \\ ${ }^{1}$ Korea Institute of Science \& Technology, Gangneung, Republic of Korea \\ ${ }^{2}$ KIST School, Korea University of Science and Technology (UST), Daejeon, Republic of Korea
}

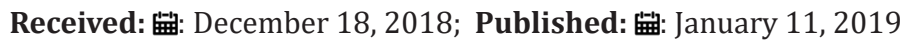

*Corresponding author: Jin Chul Kim, KIST-Gangneung Institute, Gangneung, Republic of Korea

\begin{abstract}
Psoriasis is a chronic inflammatory auto-immune disease, which causes serious skin lesions, acanthosis and parakeratosis, leaving a longlasting detrimental influence on our appearances and life quality. Though a huge scientific information regarding psoriasis-related mechanism and pathological analysis is getting accumulated over the last decades in the price of valuable experiments and efforts of many experts. However, an absence of reliable mouse model has been a major obstacle to further advances in psoriasis research. Recently, a topical application of imiquimod (IMQ), classical Toll-like Receptor (TLR)-7 agonist, has provided a strong foundation of psoriasis study on the animal model as it stimulates cutaneous phenotype similar to human psoriasis in the mouse. IMQ-mediated skin inflammation often exhibits slight divergences on human psoriasis and extensive investigations to yield more suitable psoriatic model are taking place. In this review, we summarized the recent novel breakthroughs on improving existing IMQ model and discovering a new model of psoriasis and discussed their importance on psoriasis research.
\end{abstract}

Keywords: Psoriasis; Imiquimod; Animal model; Skin inflammation; TLR

Abbreviations: IMQ: Imiquimod; TLR: Toll-like Receptor; KO: mice-Knockout mice; DC: Dendritic cell

\section{Introduction}

\section{The phenotypes of IMQ-Induced Mouse Model}

Imiquimod (IMQ), hallmark ligand of Toll-like Receptor (TLR)7 in mouse and TLR7/8 in human has been known to be protective against human papilloma virus-caused warts, actinic keratosis and basal cell carcinomas. Due to its small chemical structure and lipophilicity, it is really suitable for topical administration, thus successfully used as an ointment for the aforementioned diseases $[1,2]$. Sooner IMQ treatment appeared to be a reason for psoriasis for people who are prone to psoriasis. Since the first article of IMQinduced mouse inflammatory model was published in 2009, it emerged as one of the most prominent animal models in psoriasis study as scientist have adopted this model to their research practice and experiments. The long-term topical administration of IMQ on skin ear or shaved back skin results psoriasis-like skin inflammation, characterized by increased skin erythema and scaling, thickened epidermis, alteration of keratinocytes differentiation and recruitment of immune cells to skin, mimicking psoriatic pathogenesis in human [3,4].

\section{Pathomechanism of IMQ Induced Mouse Model}

Generally, IMQ exerts its antiviral and antitumor activity through activation of TLR7 and TLR8, intracellular receptors in macrophages and dendritic cells (DC) [5]. IMQ binding to TLR7 signals in macrophage and dendritic cells thrusts those cells to infiltrate to infection/inflammatory sites and to express pro-inflammatory cytokines and chemokines such as IL- $1 \alpha$, IL-6, IL-23, and IFN- $\alpha$, resulting in maturation of DCs and initiation of Th1/Th17 responses [6]. Moreover, IMQ treatment enhance infiltration of neutrophil, the proliferation of keratinocytes and changes in mast cells action. During the first phase of inflammation, the activation of MyD88, downstream target of TLR7/8 primarily causes the production of IL-23 from CD11c+ myeloid DCs and INF- $\alpha$ from plasmacytoid DCs to amplify inflammatory reaction and start differentiation of naive T cells via upregulation of NF- $\kappa \mathrm{B}$ and MAPK signaling $[7,8]$. In response to IL-23 secretion, $\gamma \delta \mathrm{T}$ cells predominantly release IL-17 and IL-22, which activate keratinocytes to proliferate and increase a production of chemokines and antimicrobial proteins as well as recruitment of neutrophils, thereby attracting other immune cells to maintain local inflammation.

On the other hand, IMQ also triggers keratinocyte proliferation and apoptosis through MyD88 independent pathway, possibly adenosine receptor-mediated cAMP pathway, since keratinocytes lack intracellular TLR7/8 receptor during the initial phase of inflammation and later, keratinocyte activity is regulated by IL-17 and IL-22 expression via switching on inflammasome complex to enhance level of S100 proteins and LL-37 [9,10]. All sequential 
and balanced inflammatory action of multiple cells and molecules, which is inducible by repeated administration of IMQ reach its peak after 7 days and likely to be maintained as long as if treatment continues and bear remarkable resemblance with human psoriasis, thereby making it most reliable model.

\section{Nuisances in the IMQ Model and Other Mouse Models}

]Even though, IMQ-induced mouse model mirrors major pathological traits of human psoriasis, over the time, some strain or time-dependent inconsistency were observed among experimental animals and Therefore, more efforts to improve IMQ psoriatic model are being made by applying IMQ to various KO mouse models or promoting utilization of other models. According to the Wang et al, skin inflammation on IMQ mouse model is likely to decrease after 8 days of IMQ treatment and giving IMQ to K14-VEGF KO mouse has restored chronic inflammation and retained intensity for 2 weeks, indicating that application of IMQ to KO mouse may compensate drawbacks of IMQ mouse model [11]. Strain-dependent gene expression pattern of IMQ-administered different strain mouse models, including C57BL/6 (B6), BALB/c, CD1, DBA/1, FVB/Nj, 129X1/SvJ and MOLF/EiJ has been evaluated by using an RNAsequencing technique. The significant differences have been noticed between BALB/C and B6, the most frequently used mouse strains, as genes, which are involved in DNA-replication, IL-17 stimulation, and CD8+ T cells, were upregulated, compared to CD4+ T cells and interferon signaling-related genes in B6 strain. After all, a B6 strain exhibited more accurate and consistent phenotype and expression pattern with human psoriasis [12].

Another article reported that five different transgenic psoriasis model of K5-Tie2, IMQ K14-AREG, K5-Stat3C, and K5-TGFbeta1 have been quantitatively assessed for their whole genome expression profile via microarray. While all models displayed robust correspondence to human psoriasis for morphogenesis of epidermis and keratinization-linked genes, the alterations in immune pathogenesis of psoriasis were significantly fluctuated between models, showing that each model possesses distinct expression pattern on a genomic level [13].

\section{Conclusion}

Scientific awareness and novel findings on a study of the etiology and pathogenesis for human psoriasis have been extensively accomplished since the successful practical application of IMQ to mouse as a psoriatic animal model and numerous investigations is still ongoing to reveal underlying mechanism of psoriasis based on this model. In fact, cutaneous symptoms generated by IMQ are presented remarkable similarities in regard to human psoriasis, featuring epidermal thickening, activation of Th17/Th23 cells and increased keratinocyte proliferation. Furthermore, modern analytical tools like RNA-sequencing and microarray allowed us to dig into the deep insight of molecular mechanism of IMQ and other mouse models and those novel approaches did not confirm our understanding of human psoriasis, but also gave clues on the utilization of IMQ and other mouse models. As mentioned in previous reports, administration of IMQ to B6 strain mouse generate the best-matched phenotype and genetic background than other strains and mouse models other than IMQ could provide distinct features of psoriasis, though mostly mimic characteristic of human psoriasis for cutaneous lesion development and epidermal symptoms. Therefore, IMQ model yield closest features to human psoriasis and careful selection of mouse strains and their genetic profiles before experimental planning is highly advisable.

\section{Acknowledgement}

This work is supported by an intramural grant (2E27833) from the Korea Institute of Science and Technology (KIST), Republic of Korea and the Nano Convergence Industrial Strategic Technology Development Program(2MR7230, 20000105, Development of Cosmeceutical Material Platform using Organo-Nano Complexes based on Natural Active Compounds) funded By the Ministry of Trade, Industry \& Energy (MOTIE, Korea).

\section{References}

1. Chollet JL, Jozwiakowski MJ, Phares KR, Rreiter MJ, Roddy PJ, et al. (1999) Development of a topically active imiquimod formulation. Pharm Dev Technol 4(1): 35-43.

2. Schon MP, M Schon (2007) Imiquimod: mode of action. Br J Dermatol 157(Suppl 2): 8-13.

3. Vinter H, Kragballe K, Steiniche T, Gaestel M, Iversen L, et al. (2016) Tumour necrosis factor-alpha plays a significant role in the Aldarainduced skin inflammation in mice. Br J Dermatol 174(5): 1011-1021.

4. Nadeem A, Al Harbi NO, Al Harbi MM, El Sherbeeny AM, Ahmad SF, et al. (2015) Imiquimod-induced psoriasis-like skin inflammation is suppressed by BET bromodomain inhibitor in mice through RORC/IL17A pathway modulation. Pharmacol Res 99: 248-257.

5. El Malki K, Karbach SH, Huppert G, Zayoud M, Reibig S, et al. (2013) An alternative pathway of imiquimod-induced psoriasis-like skin inflammation in the absence of interleukin-17 receptor a signaling. J Invest Dermatol 133(2): 441-451.

6. Flutter B, FO Nestle (2013) TLRs to cytokines: mechanistic insights from the imiquimod mouse model of psoriasis. Eur J Immunol 43(12): 31383146.

7. Ueyama A, Yamamoto M, Tsujii K, Furue Y, Imura C, et al. (2014) Mechanism of pathogenesis of imiquimod-induced skin inflammation in the mouse: a role for interferon-alpha in dendritic cell activation by imiquimod. J Dermatol 41(2): 135-143.

8. Rabeony H, Pohin M, Vasseur P, Petit Paris I, Jegou JF, et al. (2015) IMQinduced skin inflammation in mice is dependent on IL-1R1 and MyD88 signaling but independent of the NLRP3 inflammasome. Eur J Immunol 45(10): 2847-2857.

9. Costa S, Marini O, Bevilacqua D, DeFranco AL, Hou BD, et al. (2017) Role of MyD88 signaling in the imiquimod-induced mouse model of psoriasis: focus on innate myeloid cells. J Leukoc Biol 102(3): 791-803.

10. Schon MP, M Schon, KN Klotz (2006) The small antitumoral immune response modifier imiquimod interacts with adenosine receptor signaling in a TLR7- and TLR8-independent fashion. J Invest Dermatol 126(6): 1338-1347.

11. Wang X, J Sun, J Hu (2015) IMQ Induced K14-VEGF Mouse: A Stable and Long-Term Mouse Model of Psoriasis-Like Inflammation. PLoS One 10(12): e0145498.

12. Swindell WR, Michaels KA, Sutter AJ, Diaconu D, Fritz Y, et al. (2017) Imiquimod has strain-dependent effects in mice and does not uniquely model human psoriasis. Genome Med 9(1): 24.

13. Swindell WR, Johnston A, Carbajal S, Han GW, Wohn C, et al. (2011) Genome-wide expression profiling of five mouse models identifies similarities and differences with human psoriasis. PLoS One 6(4): e18266. 
ISSN: 2574-1241

DOI: 10.26717/BJSTR.2019.13.002347

Jin Chul Kim. Biomed J Sci \& Tech Res

(c) (i) This work is licensed under Creative BY Commons Attribution 4.0 License

Submission Link: https://biomedres.us/submit-manuscript.php

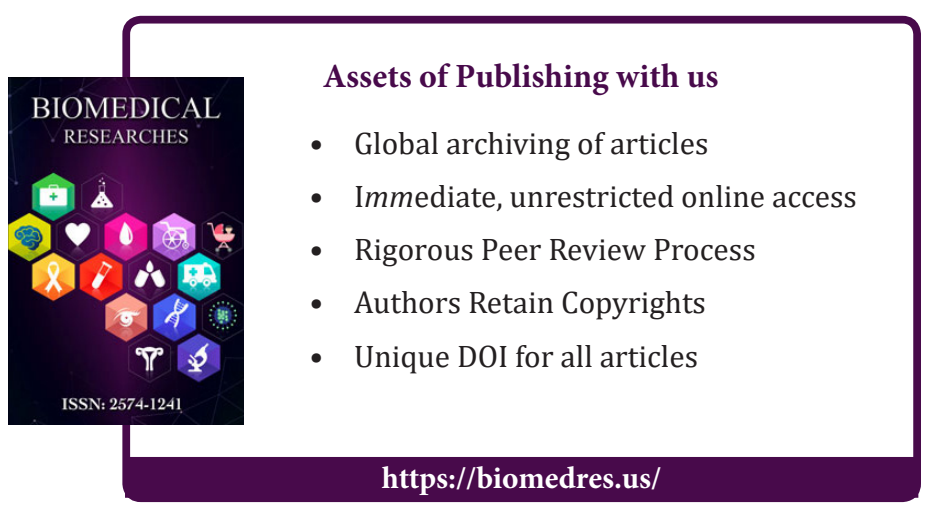

